\title{
The Investigation of Dissociation of Trifluoroacetyl Fluoride in the Field of Pulsed $\mathrm{CO}_{2}$ Laser
}

\author{
E. B. ASLANIDI, V. T. ZARUBIN and Yu. S. TURISHCHEV
}

Institute of Stable Isotopes, State Committee on the Uses of Atomic Energy, 380086 Tbilisi, USSR

(Received November 15, 1984; in final form August 13, 1985)

$\mathrm{CF}_{3} \mathrm{COF}$ dissociation channels in the pressure region from 0.2 to 4 Torr under the pulsed $\mathrm{CO}_{2}$ laser radiation are investigated. The dissociation products $\mathrm{COF}_{2}, \mathrm{C}_{2} \mathrm{~F}_{6}$, and $\mathrm{CF}_{4}$ are identified according to the infrared spectra, and their relative output is measured over the whole range of the initial substance pressures. A scheme of laserochemical reactions describing the trifluoroacetyl fluoride dissociation channels is presented. The correctness of the chosen scheme is confirmed on the basis of the elementary theory of active collisions. The measurements of $\mathrm{CF}_{3} \mathrm{COF}$ dissociation selectivity for ${ }^{18} \mathrm{O}$ and ${ }^{13} \mathrm{C}$ are given.

KEY WORDS: Multiphoton infrared dissociation; Trifluoroacetyl; isotope selectivity.

The interaction of complicated organic molecules with the infrared laser radiation has been investigated by numerous authors. ${ }^{1}$ Initial interest was aroused in particular by the demonstration that the process was isotopically selective. However, in spite of a sufficiently large amount of experimental and theoretical papers, it is not always possible to describe unambiguously the dissociation channels. The investigation of the dissociation of new compounds makes a certain contribution into this problem.

In the present paper the channels of trifluoroacetyl fluoride dissociation are investigated at the excitation by the pulsed $\mathrm{CO}_{2}$ laser. This substance can be considered as an initial material for carbon and oxygen isotopes separation. $\mathrm{CF}_{3} \mathrm{COF}$ represents a gaseous substance 
with the boiling temperature of $214 \mathrm{~K}$. The molecule structure corresponds to the $C_{s}$ symmetry group. The infrared absorption spectrum contains 15 principal vibrational bands: ${ }^{2} \quad \nu_{1}=1890 \mathrm{~cm}^{-1}, \quad \nu_{2}=$ $1340 \mathrm{~cm}^{-1}, \nu_{3}=1254 \mathrm{~cm}^{-1}, \nu_{4}=1099 \mathrm{~cm}^{-1}(\mathrm{C}-\mathrm{F}), \nu_{5}=806 \mathrm{~cm}^{-1}, \nu_{6}=$ $761 \mathrm{~cm}^{-1}, \nu_{7}=692 \mathrm{~cm}^{-1}, \nu_{8}=595 \mathrm{~cm}^{-1}, \nu_{9}=390 \mathrm{~cm}^{-1}, \nu_{10}=228 \mathrm{~cm}^{-1}$, $\nu_{11}=1214 \mathrm{~cm}^{-1}, \nu_{12}=519 \mathrm{~cm}^{-1}, \nu_{13}=426 \mathrm{~cm}^{-1}, \nu_{14}=242 \mathrm{~cm}^{-1}, \nu_{15}=$ $50 \mathrm{~cm}^{-1}$. At room temperature, $\mathrm{CF}_{3} \mathrm{COF}$ is a stable gaseous substance. The substance may stay in the reactor for several hours, which results in the high stability of the measurements.

$\mathrm{CF}_{3} \mathrm{COF}$ irradiation at room temperature was carried out by a pulsed $\mathrm{CO}_{2}$ laser with pre-ionization at the cathode. The duration of the laser pulse at its half-height was $120-150 \mathrm{~ns}$, the pulse energy was $6 \mathrm{~J}$ at the wavelength $R(16) 1075.99 \mathrm{~cm}^{-1}$, and $3 \mathrm{~J}$ at the wavelength $R(40)$ $1090 \mathrm{~cm}^{-1}$. The area of the laser beam at the inlet and outlet of the experimental reactor was approximately the same, equal to $250 \mathrm{~mm}^{2}$. The experimental reactor represents a cylindrical cell $100 \mathrm{~mm}$ long and $30 \mathrm{~mm}$ in diameter made of stainless steel. The inlet and outlet windows are closed with plane-parallel $\mathrm{NaCl}$ plates, and the seal is made of fluoroplastic with an indium spacer. The reactor was prepassivated with fluorine. The absorption spectra have been recorded by the spectrophotometer IKS-29 over the range from 4000 to $400 \mathrm{~cm}^{-1}$. To record the absorption spectra of the dissociation products at the pressure below 4 Torr, the number of experiments was increased with decreasing pressure of the working substance introduced per one experiment. The dissociation products were frozen out into the measuring reactor, and the recording of the IR absorption spectra was carried out at their constant pressure of 4 Torr ( 4 experiments for the pressure of 1 Torr in the reactor, 8 experiments for that of 0.5 Torr, etc.). The threshold $\mathrm{CF}_{3} \mathrm{COF}$ dissociation energy was $\sim 0.8 \mathrm{~J} / \mathrm{cm}^{2}$ in the spectrum maximum, at $R(20) 1078.6 \mathrm{~cm}^{-1}$.

The analysis of the IR absorption spectra of $\mathrm{CF}_{3} \mathrm{COF}$ dissociation products suggest the presence of the following substances: $\mathrm{COF}_{2}$ (absorption bands at $1929 \mathrm{~cm}^{-1}, 1243.7 \mathrm{~cm}^{-1}, 965 \mathrm{~cm}^{-1}$ ), $\mathrm{C}_{2} \mathrm{~F}_{4}$ (absorption bands at $1340 \mathrm{~cm}^{-1}$ and $1190 \mathrm{~cm}^{-1}$ ), $\mathrm{C}_{2} \mathrm{~F}_{6}$ (absorption bands at $1250 \mathrm{~cm}^{-1}$ and $1116 \mathrm{~cm}^{-1}$ ), $\mathrm{CF}_{4}$ (absorption bands at $1280 \mathrm{~cm}^{-1}$ and $2174 \mathrm{~cm}^{-1}$ ).

To carry out the quantitative analysis of the dissociation products, the intensities of pure $\mathrm{COF}_{2}$ and $\mathrm{C}_{2} \mathrm{~F}_{4}$ absorption as the functions of the pressure in the experimental reactor were measured. Figure 1 


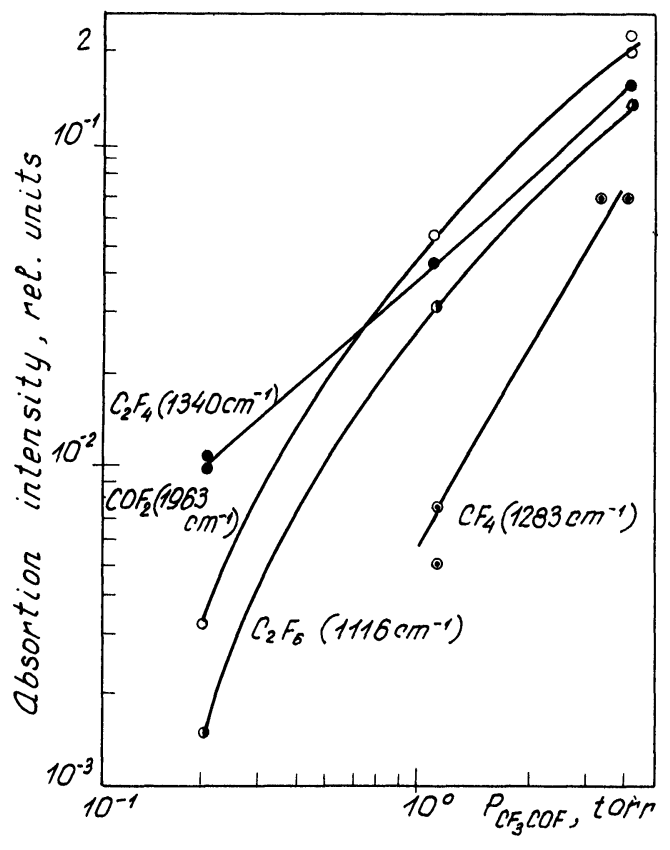

FIGURE 1 The dependence of the output of $\mathrm{CF}_{3} \mathrm{COF}$ dissociation products on its pressure $\left(\nu=1082.3 \mathrm{~cm}^{-1} ; E=6 \mathrm{~J}\right)$.

shows the experimentally observed dependences. The intensities of the spectral lines of all dissociation products are normalized to the same scale of relative units.

On the basis of the obtained data, we can come to the following conclusions: in the pressure region below 0.6 Torr, the reaction product is mainly $\mathrm{C}_{2} \mathrm{~F}_{4}$, while the amount of $\mathrm{C}_{2} \mathrm{~F}_{6}$ and $\mathrm{COF}_{2}$ is small. At the pressure above 1 Torr, the production rates of all the substances become equal and, in any case, remain constant up to 4 Torr (the pressures above 4 Torr were not investigated). Besides, at the pressure of 1 Torr, $\mathrm{CF}_{4}$ appears among the products. The obtained data have allowed us to suggest that the formation of $\mathrm{CF}_{3} \mathrm{COF}$ dissociation 
products proceeds according to the following mechanism:

$$
\mathrm{CF}_{3} \mathrm{COF}+n h \nu \quad\left\{\begin{array}{l}
\mathrm{CF}_{3} \mathrm{COF}^{*} \rightarrow \mathrm{C}_{2} \mathrm{~F}_{4}+\mathrm{O} \\
\mathrm{CF}_{3} \mathrm{COF}^{*}+M \rightarrow \mathrm{COF}+\mathrm{CF}_{3}+M
\end{array}\right.
$$

i.e., the initial molecule decomposition may proceed by two channels.

Then the following reactions occur:

$$
\begin{aligned}
\mathrm{COF}+\mathrm{COF} & \rightarrow \mathrm{COF}_{2}+\mathrm{CO} \\
\mathrm{CF}_{3}+\mathrm{CF}_{3} & \rightarrow \mathrm{C}_{2} \mathrm{~F}_{6} \\
\mathrm{CO}+\mathrm{O} & \rightarrow \mathrm{CO}_{2} \\
\mathrm{CF}_{3}+\mathrm{O} & \rightarrow \mathrm{COF}_{2}+\mathrm{F}
\end{aligned}
$$

The interaction of $\mathrm{COF}$ and $\mathrm{CF}_{3}$ radicals with the initial molecule may serve as a possible channel of $\mathrm{C}_{2} \mathrm{~F}_{6}$ and $\mathrm{COF}_{2}$ formation. The increase of $\mathrm{CF}_{3} \mathrm{COF}$ pressure should lead to the dependence observed in the experiment (see Figure 1). To obtain the answer concerning the role of such processes, the buffer gas ( $\mathrm{He}$ or $\mathrm{Xe}$ ) was added to $\mathrm{CF}_{3} \mathrm{COF}$. The increase of the buffer pressure up to 40 Torr did not lead to any qualitative changes of the dependence obtained before. Therefore, we may conclude that the role of such secondary processes is slight.

To confirm the scheme presented above, we apply the elementary theory of active collisions. It is well-known ${ }^{3}$ that this theory is based on the supposition that bimolecular reactions are realized at the collision of two molecules. The reaction rate is proportional to the collision number in a unit volume during a unit of time. The molecule decomposition proceeds through an activated complex representing a certain instantaneous state of the active molecule, the excitation energy being concentrated at certain degrees of freedom and, finally, leading to the dissociation.

Let us consider the dissociation of the initial molecule $A$ located in the thermal reservoir consisting of the molecules $M$, under the action of the laser radiation:

$$
\begin{aligned}
& A+n h \nu \stackrel{K_{i}}{\longrightarrow} A^{*} \\
& A^{*} \stackrel{K_{2}}{\longrightarrow} B+C
\end{aligned}
$$




$$
\begin{aligned}
& A^{*}+M \stackrel{K_{3}}{\longrightarrow} D+E \\
& A^{*}+M \stackrel{K_{4}}{\longrightarrow} A+M
\end{aligned}
$$

where $B, C, D$ and $E$ are the dissociation products.

We may apply the stationarity principle to the activated molecule $A^{*}$ serving as an unstable intermediate complex:

$$
\frac{d\left[A^{*}\right]}{d t}=k_{1}[A]-k_{2}\left[A^{*}\right]-k_{3}\left[A^{*}\right][M]-k_{4}\left[A^{*}\right][M]=0
$$

hence,

$$
\left[A^{*}\right]=\frac{k_{1}[A]}{k_{2}+\left(k_{3}+k_{4}\right)[M]}
$$

The observed decomposition rate $[A]$ is determined from the expression

$$
-\frac{d[A]}{d t}=k_{2}\left[A^{*}\right]+k_{3}\left[A^{*}\right][M]
$$

Substituting the concentration $\left[A^{*}\right]$ from the previous equation, we obtain

$$
-\frac{d[A]}{d t}=k_{2} \frac{k_{1}[A]}{k_{2}+\left(k_{3}+k_{4}\right)[M]}+k_{3} \frac{k_{1}[A][M]}{k_{2}+\left(k_{3}+k_{4}\right)[M]}
$$

Two limiting cases are possible depending on the initial substance pressure.

1. At low pressures

$$
\begin{gathered}
k_{2} \gg\left(k_{3}+k_{4}\right)[M] \\
-\frac{d[A]}{d t}=k_{1}[A]+k_{1} \frac{k_{3}}{k_{2}}[A][M]
\end{gathered}
$$

The analysis of the obtained expression shows that at small $[A]$ and $[M]$, the decomposition of $[A]$ occurs in the first order fashion. The increase of the initial substance pressure leads to the primary decomposition in the second order fashion (the second term makes the main contribution). 
2. At high pressures

$$
\begin{aligned}
-\frac{d[A]}{d t}= & k_{2} \frac{\left(k_{3}+k_{4}\right)[M] \gg k_{2}}{\left(k_{3}+k_{4}\right)[M]}+\frac{k_{1} k_{3}[A][M]}{\left(k_{3}+k_{4}\right)[M]} \\
= & \frac{k_{1} k_{2}}{k_{3} k_{4}} \frac{[A]}{[M]}+\frac{k_{1} k_{3}}{k_{3}+k_{4}}[A]
\end{aligned}
$$

In case of the pure substance dissociation $[A]=[M]$, and $-d[A] / d t=k^{\prime}[A]+b$ is a first-order reaction where

$$
k^{\prime}=\frac{k_{1} k_{3}}{k_{3}+k_{4}}, \quad b=\frac{K_{1} K_{2}}{K_{3}+K_{4}}
$$

The above schematic representation of the reaction confirms the validity of the above scheme (1)-(4).

The production rates of all the products become equal at the pressure of 0.5 Torr. In the general case, the expression of the reaction rate is the following:

$$
\begin{array}{ll}
w_{1}=k_{1}^{\prime}[A] & \text { in the first order } \\
w_{2}=k_{2}^{\prime}[A][M] & \text { in the second order }
\end{array}
$$

$\mathrm{CF}_{3} \mathrm{COF}$ decomposition is experimentally observed by the output of laserochemical reaction products $\mathrm{C}_{2} \mathrm{~F}_{4}, \mathrm{COF}_{2}$ and $\mathrm{C}_{2} \mathrm{~F}_{6}$. Now we show that the final products can unambiguously define the initial compound decomposition in the case under consideration. We derive the production of $\mathrm{C}_{2} \mathrm{~F}_{6}$ out of $\mathrm{CF}_{3} \mathrm{COF}$ on the basis of the notion of two subsequent second order reactions:

$$
\begin{gathered}
A^{*}+M \stackrel{K_{3}}{\longrightarrow} D+E \\
D+D \stackrel{K_{5}}{\longrightarrow} N
\end{gathered}
$$

The kinetics of the process under consideration will be described by the following equations:

$$
\begin{aligned}
& \frac{d[N]}{d t}=k_{5}[D]^{2} \\
& \frac{d[D]}{d t}=k_{3}\left[A^{*}\right][M]-k_{5}[D]^{2}
\end{aligned}
$$


According to the stationarity condition,

$$
\frac{d[D]}{d t}=0
$$

then

$$
\begin{aligned}
& {[D]^{2}=\frac{k_{3}}{k_{5}}\left[A^{*}\right][M]} \\
& \frac{d[N]}{d t}=k_{3}\left[A^{*}\right][M]
\end{aligned}
$$

Hence, the production rate of $\mathrm{C}_{2} \mathrm{~F}_{6}$ is determined by $\mathrm{CF}_{3} \mathrm{COF}$ dissociation rate.

The parallel reactions (5) and (6) are competing with respect to the oxygen atoms. The reaction (6) is the least probable, since it should initiate the subsequent reaction

$$
\mathrm{CF}_{3}+\mathrm{F} \longrightarrow \mathrm{CF}_{4}
$$

The absence of $\mathrm{CF}_{4}$ among the products at low pressures of the initial substance confirms the above supposition.

$\mathrm{CF}_{4}$ formation at $\mathrm{CF}_{3} \mathrm{COF}$ pressure above 1 Torr may be, generally, described through several mechanisms. However, the experimental data do not allow us to describe the observed situation unambiguously. It should be noted that $\mathrm{CF}_{4}$ formation is observed at the dissociation of numerous molecules (the pressure exceeding several Torr) containing $\mathrm{CF}_{3}$ groups $\left(\mathrm{CF}_{3} \mathrm{COCF}_{3}, \mathrm{CF}_{3} \mathrm{OF}\right.$, etc. $)$. Therefore, we may believe that the mechanism should be of a sufficiently general character, i.e., it should be described by an equation of the type of Eq. (7).

A pure $\mathrm{CF}_{3} \mathrm{COF}$ of natural isotopic composition was used (at pressure 1 Torr) for preparing the dissociation products isotopically modified in ${ }^{13} \mathrm{C}$ and ${ }^{18} \mathrm{O}$. Spectral dependence of the degree of $\mathrm{COF}_{2}$ enrichment in the lasing frequency range from 980 to $1072 \mathrm{~cm}^{-1}$ was determined. The best results at the $\mathrm{P}(30) 1037.4 \mathrm{~cm}^{-1}$ line were as follows: $6 \%$ for ${ }^{13} \mathrm{C}$ and $0.53 \%$ for ${ }^{18} \mathrm{O}$ which corresponds to a selectivity 10 for both the isotopes. Isotopic analyses were done on the mass spectrometer $\mathrm{MI}-1201$ on the mass lines $66,67,68\left(\mathrm{COF}_{2}^{+}\right)$ and 116, 117, $118\left(\mathrm{CF}_{3} \mathrm{COF}^{+}\right)$. The $\mathrm{C}_{2} \mathrm{~F}_{6}$ enrichment in ${ }^{13} \mathrm{C}$, measured in terms of $\mathrm{C}_{2} \mathrm{~F}_{5}^{+}$ions at pressures $\sim 1$ Torr represents the enrichment for $\mathrm{COF}_{2}$. According to the dissociation mechanism proposed and 
Eqs. (1) and (2) the highest enrichment in ${ }^{13} \mathrm{C}$ should be expected in tetrafluoroethylene resulting from the monomolecular decay of the initial material. The $\mathrm{C}_{2} \mathrm{~F}_{4}$ produced directly in the mass spectrometer from the residual $\mathrm{CF}_{3} \mathrm{COF}$ however prevents from isolating the mass lines of laser dissociation products.

\section{CONCLUSIONS}

It is shown that the $\mathrm{CF}_{3} \mathrm{COF}$ dissociation depending on its pressure in the field of a pulsed $\mathrm{CO}_{2}$ laser light can be described by a reaction as a result of active collisions of particles having some adequate energy. Low dissociation threshold and selectivity $\sim 10$ with the dissociation yield $\sim 8 \%$ allow trifluoroacetyl fluoride to be considered as rather a promising material for oxygen isotopes separation.

\section{References}

1. V. N. Bagratashvili, V. S. Letokhov, A. A. Makarov and E. A. Ryabov, Multi-Photon Processes in Molecules in the Infrared Laser Field (VINITI, Moscow, 1980).

2. C. V. Berney, Spectrochim. Acta 27A, 663-672 (1971).

3. V. N. Kondratyev and E. E. Nikitin, The Kinetics and the Mechanism of the GaseousPhase Reactions (Nauka, Moscow, 1974). 